\title{
LES RADIOPROTECTEURS CHIMIQUES
}

\author{
Y. ROBBE* \\ (Manuscrit regu le 9 octobre 1972 )
}

Si les possibilités énergétiques de l'atome sont immenses, si son utilisation à des fins pacifiques peut modifier radicalement l'évolution de la civilisation, cette ère prodigieuse pose de façon aiguë le problème de la protection de l'homme. Il est donc vital que la thérapeutique puisse disposer dans un avenir prochain de radioprotecteurs ou d'associations de substances capables d'éliminer, ou tout au moins de minimiser, les effets nocifs, sinon mortels, des irradiations.

Quels risques, existe-t-il, pour l'être vivant dans l'âge atomique?

- tout d'abord, des effets somatiques non cancérogènes [ $\mathrm{r}$ à 6 ] qui seront plus ou moins précoces et sévères, suivant la dose reçue;

- puis, l'apparition, souvent tardive, des effets somatiques cancérogènes qui entraînent une létalité différée. Ici la gravité du mal ne dépendrait pas de la dose d'énergie, car, en fait, il n'y aurait pas de dose seuil de sécurité. Toute irradiation aussi faible soit-elle, serait capable d'engendrer des cancers : la probabilité étant toujours faible, mais jamais nulle, augmenterait avec l'importance de la dose reçue $[3,6]$;

- enfin, des effets héréditaires induits par les mutations [7, 8]. Comme les radiations augmentent artificiellement la fréquence de ces mutations, celles-ci marqueront leur empreinte sur la race humaine dans les générations futures.

Depuis 1945, l'homme s'est attaché non seulement à rechercher les meilleurs moyens de protection, à partir d'écrans (béton, plomb et autres matériaux) et dispositifs assurant un véritable blindage antiradiations, mais il a essayé également de trouver des susbstances capables d'éviter la maladie des rayons.

La première lueur d'espoir est apparue en 1948 lorsque LATARJET et EpHraTI [9] ont montré qu'il était possible de protéger un bactériophage contre les radiations, par des substances chimiques tels l'acide thioglycolique, le glutathion, le tryptophane, la cystéine et la cystine.

Un an plus tard, la faculté de diminuer l'effet nocif des radiations chez la souris était mise en évidence, en utilisant le cyanure [10], la cystéine [I I ] et la thiourée [1 2]. Mais c'est BACQ et ses collaborateurs qui, en mettant en lumière l'action remarquable de la cystéamine [13], montraient que la radioprotection n'était point une utopie chez les mammifères.

* Laboratoire de chimie organique pharmaceutique (Dir. Professeur R. Granger), Faculté de Pharmacie et Institut européen de Pharmacie industrielle de Montpellier. Travail effectué avec le concours de la D.R.M.E. (Pharmacien chimiste en chef de $\mathrm{I}^{\mathrm{re}}$ classe JÉzÉQueL).

RADIOPROTECTION, VOL. 7 - $\mathrm{N}^{\circ} 4$ 
Pendant longtemps, il parut pratiquement impossible de trouver des substances possédant individuellement une efficacité supérieure à la cystéamine. Mais la découverte de nombreuses molécules aux effets importants et, en particulier, des $\omega$-amino alkyl amino alkyl-S phosphorothioates [14], a ouvert de nouveaux horizons dans la recherche des radioprotecteurs.

La radioprotection nous fait entrevoir des possibilités nouvelles qui se font jour au fur et à mesure que se développent les connaissances actuelles des mécanismes de l'action des radiations, ainsi que des mécanismes de radioprotection.

Il est admis actuellement que les effets nuisibles des radiations sont les conséquences de :

- l'action directe du quantum d'énergie $\mathrm{h} v$ au niveau des molécules essentielles, telle l'ADN;

- de l'action indirecte qui amplifie les réactions primaires dues aux radicaux libres produits par la radiolyse de l'eau, constituant essentiel des organes vivants (voir schéma).

Mais les différents auteurs ne donnent pas la même importance à l'action directe (théorie de la cible) et à l'action indirecte dans les cellules [ [ 19 à 22]

\section{MÉCANISME DE LA RADIOPROTECTION}

Le phénomène de radioprotection est universel; il n'est pas limité seulement aux êtres vivants, car les physico-chimistes l'ont observé sur des polymères de synthèse tel le polyméthacrylate ou sur des substances naturelles comme les acides nucléiques et les protéines $[16,17,18]$. La majorité des spécialistes est d'accord : l'action des protecteurs chimiques doit se situer au niveau des phénomènes physico-chimiques prenant naissance lors de l'irradiation.

Plusieurs hypothèses ont été avancées pour expliquer le phénomène de la radioprotection par certaines substances.

\section{A - Protection PAR des méCANismes RadiochimiQues}

Les agents radioprotecteurs du type aminothiol peuvent agir à trois niveaux différents pour s'opposer à l'action des radiations sur la molécule biologique $\mathrm{MH}[22]$.

\section{I - La radioprotection serait due au piégeage des radicaux libres (niveau I du schéma)}

Alexander et BACQ ont proposé cette théorie relative au piégeage des radicaux libres, formés lors de l'irradiation, par les radioprotecteurs soufrés [23, 24].

Les dérivés sulf hydrylés (PSH), comme la cystéine, la cystéamine, la mercaptoéthylguanidine (MEG) et l'aminoéthylisothiourée (ou AET) qui se transformeraient en MEG par réarrangement (bien que ceci soit contesté) [25], peuvent réagir avec les radicaux et les molécules provenant de la radiolyse de l'eau en 
présence d'oxygène $\left(\mathrm{H}^{*}, \mathrm{HO}^{\circ}, \mathrm{HO}_{2}^{*}\right.$ et $\left.\mathrm{H}_{2} \mathrm{O}_{2}\right)$ pour les neutraliser et les empêcher d'atteindre les molécules radiosensibles [1, 26].

Le radioprotecteur peut réagir également avec la molécule altérée $\mathrm{M}^{*}$ ou hydroperoxydée $\mathrm{M} \mathrm{O}_{2}^{*}[27]$ pour redonner la forme initiale $\mathrm{M}: \mathrm{H}$ dans le premier cas, et un composé stable $\mathrm{MOOH}$ dans le deuxième cas, brisant ainsi la chaîne de réactions qui aurait détruit d'autres molécules du substrat biologique.

Différentes considérations font penser à certains auteurs que ce mécanisme existe, mais qu'il joue un rôle quantitativement mineur chez les mammifères [ 23 , 28].

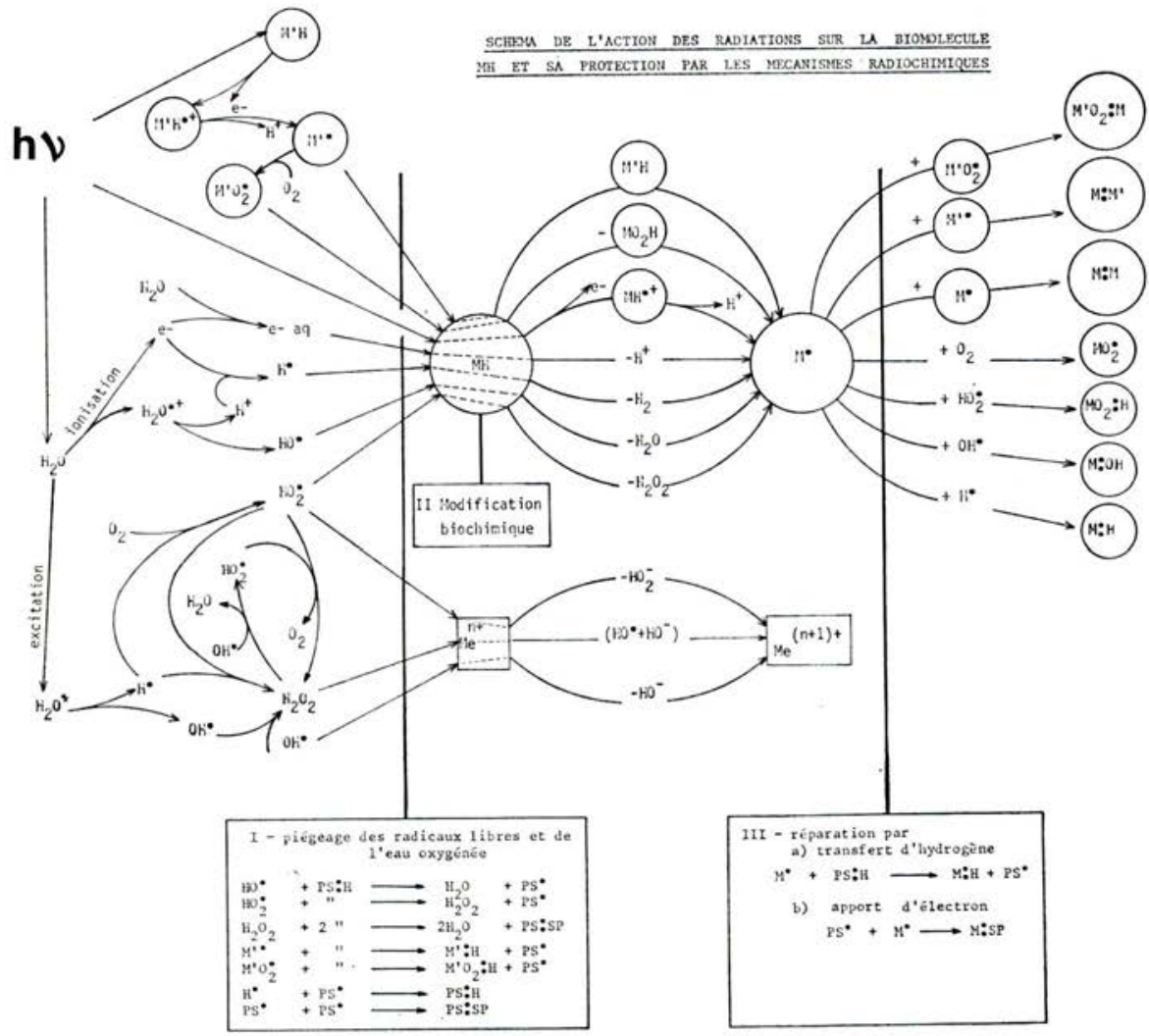

\section{2 - La radioprotection serait due à des modifications chimiques qui changeraient la radio- sensibilité intrinsèque des biomolécules (niveau II du schéma)}

La protection d'une molécule par modification chimique est particulièrement efficiente si sa fonction biologique dépend de l'intégrité d'un groupement fonctionnel qui peut être couvert ou modifié réversiblement [22].

Eldjarn et PrHL [ 29 à 32 ] ont émis l'hypothèse attrayante que l'effet de radioprotection des dérivés sulfurés serait dû à la formation de disulfures mixtes.

vOL. $7-\mathrm{N}^{0} 4$ 
Ils ont démontré que des composés telles la cystéamine et la cystamine forment des disulfures mixtes avec les protides de structure MSSM et MSH porteurs de ponts disulfures S-S ou de fonction thiol SH.

Ce mécanisme de protection est schématisé par les réactions suivantes :

$$
\begin{gathered}
\mathrm{MSSM}+\mathrm{HS}-\mathrm{CH}_{2}-\mathrm{CH}_{2} \mathrm{NH}_{2} \rightarrow \mathrm{MSS}-\mathrm{CH}_{2}-\mathrm{CH}_{2}-\mathrm{NH}_{2}+\mathrm{MSH} \\
\mathrm{MSH}+\mathrm{HS}-\mathrm{CH}_{2}-\mathrm{CH}_{2}-\mathrm{NH}_{2} \rightarrow \mathrm{MSS}-\mathrm{CH}_{2}-\mathrm{CH}_{2}-\mathrm{NH}_{2}+{ }_{2} \mathrm{H}^{\cdot}\left(\text { ou } 2 \mathrm{e}+{ }_{2} \mathrm{H}^{+}\right. \text {) }
\end{gathered}
$$

Selon cette hypothèse, ces disulfures mixtes formés temporairement devraient rendre moins vulnérable aux radiations la macromolécule originale tout en lui gardant ses caractéristiques fonctionnelles et la possibilité de se régénérer.

Jellum [33], Kollmann, Shapiro et Martin [34] ont d'ailleurs montré que la cystamine et d'autres diamino disulfures peuvent se lier à l'ADN " in vitro » au niveau de ses charges positives et le protéger contre les changements physiques induits par les radiations.

Mais ces liaisons sont-elles bien responsables de la protection?

3 - La radioprotection serait engendrée par des substances capables de réparer immédiatement les biomolécules lésées par transfert d'un atome d'bydrogène ou d'un électron (niveau III) du schéma)

Si la lésion au niveau de la biomolécule correspond à la perte d'un atome d'hydrogène, les radioprotecteurs du type aminothiol pourront la réparer instantanément par un transfert d'hydrogène $\left(\mathrm{H}^{*}\right)$ ou $\left(\mathrm{e}^{-}+\mathrm{H}^{+}\right)$(voir tableau).

Les aminothiols qui auront déjà fourni un atome d'hydrogène (PS') seront encore en mesure de réagir avec les biomolécules sulfhydrylées radicalisées pour donner des disulfures mixtes.

Les aminothiols agiront donc ici en apportant un électron, remplaçant l'électron expulsé par les rayons ionisants [35].

Il est très vraisemblable que d'autres molécules telles la sérotine [36], les phénothiazines (cité dans [37]), soient capables de transférer des électrons.

\section{B - Protection par des méCANISMes bIOChIMIQUeS ET PHYSIOlogiQues}

Plusieurs mécanismes biochimiques et physiologiques ont été invoqués.

\section{I - La radioprotection serait le résultat d'un choc biochimique}

Les thiols et disulfures radioprotecteurs induisent des effets biochimiques et physiologiques chez les mammifères, que BACQ et AleXANDER ont désignés sous le nom de "choc biochimique " [23, 38 à $4 \mathrm{I}]$.

Si l'on injecte à forte dose un radioprotecteur soufré (thiol ou disulfure) chez un animal non irradié, on constate que la presque totalité du radioprotecteur est fixée sur les protéines au bout de quelques minutes. Les molécules de disulfures sont réduites très rapidement. La fixation sur les protéines entraîne des troubles de l'équilibre endocellulaire entre les $\mathrm{SH}$ libres et combinés, ainsi que la 
régulation du potentiel d'oxydoréduction. Il s'ensuit une altération de la perméabilité des structures intracellulaires et mitochondriales par suite de la fixation du dérivé soufré sur les protéines et autres macromolécules [23] et une inhibition du métabolisme des glucides, de la synthèse des acides nucléiques, et des protéines. La division cellulaire est également retardée ou inhibée.

D'autres recherches de BACQ ont révélé que la cystéamine administrée " per os ») et de façon continue, à la souris irradiée à faible dose pendant un temps très long, n'a pas d'effet radioprotecteur.

Dans cette expérience on constate que la suppression du choc biochimique entraîne l'abolition de la protection [23].

\section{2 - La radioprotection proviendrait de la libération d'agents radioprotecteurs endogènes}

REvesz et Modig ont suggéré que la protection après administration de radioprotecteurs soufrés, telle la cystéamine, pourrait être due dans une large mesure à la libération de glutathion réduit (GSH), à partir de certaines protéines présentant des liaisons disulfures avec le glutathion :

$$
\text { ac. aminés-S-S-G + PSH } \rightarrow \text { ac. aminés -S-S-P + GSH [42 à 44] }
$$

\section{3 - La radioprotection serait due à l'augmentation de la radiorésistance cellulaire [22]}

La radiosensibilité des cellules dépend de plusieurs facteurs, un des facteurs essentiels étant l'âge de la cellule au moment de l'exposition : en effet, les cellules sont en général très sensibles pendant la mitose et dans le stade $G_{2}$, sensibles pendant la période $G_{1}$, et beaucoup moins pendant la phase $S$ [45 à 47].

SinClair a suggéré que les fluctuations des concentrations en substances sulfhydrylées non protéiniques dans la cellule peuvent être à la base des modifications de la radiosensibilité observées en fonction de l'âge.

Il semble évident que les changements métaboliques et structuraux au cours du cycle de la cellule affectent la radiosensibilité de celle-ci.

De même, il est concevable que l'administration d'un agent protecteur puisse induire des changements dans la cible ou dans l'environnement immédiat, changements susceptibles de masquer les sites radiosensibles importants ou de les exposer à un degré moindre.

\section{4 - La radioprotection serait due à une augmentation des restaurations}

Il est possible que la réduction par un radioprotecteur des dommages dus aux radiations se fasse par un mécanisme de restauration $[48,49]$.

Quelques expériences de Gourier et coll. mettent en évidence une réparation significative dans le foie du rat $[39,50,51]$.

Lorsque les rats ont subi une hépatectomie partielle, la régénération de la synthèse de l'ADN est précédée par une augmentation de l'activité de quelques enzymes telles l'ARN polymérase, la thymidine kinase et la thymidylate kinase. Ces augmentations sont dues à la synthèse “ de novo » des enzymes.

- Quand les rats sont irradiés à $\mathrm{I}$ ooo $\mathrm{R}$ avant l'hépatectomie, l'augmentation normale de l'activité des enzymes est diminuée et retardée.

vOL. $7-\mathrm{N}^{\mathrm{O}} 4$ 
- Si l'AET est administrée une heure avant l'irradiation et si l'hépatectomie partielle est réalisée 24 heures après, on n'observe pas de protection et la synthèse enzymatique est inhibée.

- Par contre, si l'AET est injectée une heure avant l'irradiation et l'hépatectomie partielle effectuée 48 heures plus tard, on constate la protection.

Ces expériences font penser que la protection nécessite un temps considérable pour se manifester et que celle-ci est réalisée par un mécanisme de restauration.

\section{C - L'irradiation exalte L'ACtivité des hydrolases ET Celle-Ci Serait INHIBÉE PAR LES RADIOPROTECTEURS}

DANYSz et ses collaborateurs ont constaté que l'irradiation augmente l'activité de plusieurs hydrolases et notamment celle des enzymes protéolytiques : cathepsine et phosphatase acide, avec comme conséquence directe l'accroissement des inhibiteurs endogènes (les haptoglobines) ainsi que l'abaissement du niveau des substrats, tels que les protéines ou les acides nucléiques [35, 52 à 54].

Si l'on administre au rat un inhibiteur des enzymes protéolytiques, tel que l'acide amino-4 méthyl-2 benzoïque (PAMBA), on observe une diminution de l'accroissement du taux des haptoglobines au cours des deux premiers jours après l'irradiation.

DANYSZ et coll. ont énoncé une hypothèse selon laquelle la destruction des membranes de la cellule et des structures subcellulaires par les irradiations faciliterait la pénétration des inhibiteurs sur les enzymes et le processus de l'inhibition : ce qui plaide en sa faveur, c'est le taux des inhibiteurs plus élevé dans les tissus des animaux irradiés. Ainsi, le niveau de l'acide $\varepsilon$-amino caproïque ou celui de l'acide amino-4 méthyl-2 benzoïque est plus augmenté dans le foie des animaux irradiés, et, en particulier, dans la fraction mitochondriolysosomyale, que chez les animaux témoins [35, 54 à 58 ].

Il n'en demeure pas moins que l'utilisation des antifibrinolytiques, aux doses inhibant complètement l'accroissement de la protéolyse après irradiation, n'exerce qu'une influence peu notable sur la survie des animaux irradiés. DANYsz explique ce fait par les hypothèses suivantes : la libération des enzymes après irradiation " enzyme release " et le processus d'autodestruction de la cellule jouent un rôle moins important dans le syndrome des rayons que celui prévu par BACQ et Alexander.

\section{D - LA RADIOPROTECTION SERATT LA CONSÉQUENCE DE LA CHÉLATION DE CERTAINS IONS $[59,60]$.}

Cette hypothèse découle de la théorie de l'action indirecte.

Les peroxydes organiques formés lors de l'irradiation sont capables d'oxyder les ions métalliques de manière souvent irréversible. Ainsi, l'ion cuivreux de la cytochrome oxydase des mitochondries, dès lors qu'il est oxydé, est incapable d'entrer en réaction avec l'oxygène.

$$
\text { Enzyme } \mathrm{Cu}^{+} \frac{\mathrm{h} v}{\text { (peroxydes organiques) }} \rightarrow \text { enzyme } \mathrm{Cu}^{++}
$$


Certains chélateurs, susceptibles d'atteindre les mitochondries, agiraient en fixant réversiblement l'ion cuivreux de la cytochrome oxydase par exemple, le complexe ainsi formé pouvant le protéger contre les radiations.

Cette théorie a été parfois évoquée pour expliquer le mode d'action de quelques aminothiols, du glutathion, des dithiocarbamates, des dithiols, etc.

\section{E - LA RADIOpRotection SERAIT LE RÉSUltat DES EFFETS ANTIMITOTIQUES}

Pour quelques auteurs, l'activité radioprotectrice de la cystéine serait liée à son pouvoir antimitotique (cité dans [6I]).

Certaines substances, d'ailleurs antimitotiques, comme la colchicine, ont un pouvoir radioprotecteur. Mais là encore, il est difficile de généraliser.

\section{F - LA RADIOPROTECTION SERAIT LE RÉSULTAT D'UNE HYPOXIE OU D'UNE ANOXIE}

Cette théorie, la plus ancienne, toujours valable, est basée sur plusieurs faits :

I - L'intensité des effets des radiations $\mathrm{X}$ ou $\gamma$ est d'autant plus grande que la pression partielle d'oxygène dans le système irradié est plus élevée [62].

Cet " effet oxygène " peut être observé aussi bien chez les bactéries que chez les plantes et les mammifères $\left[6_{3}, 6_{4}, 6_{5}\right]$.

Ainsi, chez les animaux respirant un air pauvre en oxygène, on peut observer que la réduction de la pression partielle de ce gaz dans les cellules et les tissus diminue la sévérité des lésions et augmente les effets radioprotecteurs de certains agents chimiques [1].

2 - Certaines substances capables de diminuer la tension d'oxygène dans le milieu de culture ou de provoquer une hypoxie ou une anoxie chez les mammifères par différents mécanismes, sont également susceptibles de protéger contre les radiations $[16,66]$.

Ainsi chez les mammifères, l'injection de fortes doses de cystéine, de cystéamine, de cystamine (dérivé oxydé de la cystéamine ne consommant pas d'oxygène) ou d'histamine provoquent une anoxie par ralentissement circulatoire, le degré de saturation du sang veineux en oxygène pouvant être abaissé, celui du sang artériel restant normalement saturé en général [16]. Dans le cas de l'histamine, le ralentissement circulatoire est provoqué par la vasodilation et la stagnation du sang $[23]$.

En ce qui concerne la p-amino propiophénone [r, 67 à 70$]$, les cyanures et les nitriles, il est généralement accepté que leur action protectrice est due à leur capacité de diminuer la pression partielle d'oxygène plus qu'à celle de former de la méthémoglobine.

Les substances anoxémiantes par d'autres mécanismes peuvent révéler un effet radioprotecteur intéressant. C'est le cas de la morphine, inhibant les centres respiratoires, de l'adrénaline et de la noradrénaline agissant par vasoconstriction artériolaire [23].

3 - La formation des radicaux libres est augmentée en présence d'oxygène.

Ceci montre clairement le rôle important de l'oxygène et des oxydations dans les dommages causés par les radiations.

vOL. $7-\mathrm{N}^{\circ} 4$ 
G - LA RADIOPROTECTION PROVIENDRAIT DE L'ACTIVATION PAR LES RADIOPROTECTEURS $[37,7 \mathrm{I}$ ] DE LA VOIE DES PENTOSES (VOIE OXYDATIVE DIRECTE : vOD)

Lors de l'irradiation, la voie oxydative directe est inhibée. Il faut rappeler ici sommairement le rôle métabolique de la VOD pour comprendre les répercussions qu'entraînent son inhibition.

En effet, la VOD a deux fonctions essentielles.

I - Syntbèse du ribose et du désoxyribose

Le ribose est formé à partir du glucose par perte de quatre atomes d'hydrogène, puis décarboxylation avec pour intermédiaire le ribulose phosphate.

Le ribose est ensuite transformé en désoxyribose en présence de NADPH $+\mathrm{H}^{+}$, et, par suite, prend part à la synthèse de l'ADN et de l'ARN.

\section{2 - Réduction du NADP en NADPH + $\mathrm{H}^{+}$}

Cette dernière molécule NADPH joue un rôle considérable et est indispensable dans de nombreuses réactions de réduction telles :

- la transformation de l'acide folique en acide tétrahydrofolique;

- la biosynthèse des lipides;

- la kiosynthèse de certains aminoacides (tyrosine, phénylalanine);

- la réduction des liaisons disulfures (on connaît le rôle du groupe SH dans l'activation de certaines déshydrogénases et dans la perméabilité membranaire);

- le mécanisme de détoxication;

- la réduction de la méthémoglobine;

- le maintien du potentiel d'oxydoréduction.

La VOD est en outre indispensable à la croissance et à la régénération des tissus.

L'inhibition de la VOD provoque, dans les tissus irradiés, des tendances inverses à celles dont ils ont besoin pour se régénérer. Un bon radioprotecteur devra donc activer la VOD.

Quelques objections ont pu être faites à la plupart de ces théories; aucune à elle seule ne peut expliquer le phénomène de radioprotection. Tout conduit donc à penser que les radioprotecteurs peuvent agir par des mécanismes différents ou par plusieurs mécanismes simultanés.

\section{MESURE DE LA RADIOPROTECTION OBTENUE AVEC LES DIFFÉRENTES SUBSTANCES}

Cette mesure consiste à évaluer l'efficacité des diverses substances étudiées en radioprotection.

Dans ce but, les chercheurs ont été amenés à mettre au point différents tests sur un " matériel») très varié allant des molécules simples aux organismes vivants $[16,30,72]$. 
Les méthodes d'évaluation peuvent être divisées en tests in vitro et in vivo; les premiers doivent être différenciés à leur tour en tests chimiques $[26,73$ à 76$]$ et en tests biologiques [73, 77 à 82$]$.

Les tests " in vitro " [ $1,16,83$ à 86] sont sans aucun doute d'un grand intérêt, en particulier lorsqu'il s'agit de suivre les réactions, vis-à-vis du rayonnement, des cellules isolées en présence d'un radioprotecteur. Mais les résultats obtenus, même sur la cellule isolée, ne peuvent pas être extrapolés à l'animal.

Parmi les tests " in vivo " la mesure du taux de survie est la méthode la plus employée et peut-être la plus valable pour mesurer l'efficacité d'un radioprotecteur.

Ce test s'applique principalement à la source irradiée en présence ou en absence de radioprotecteur. Il consiste à administrer le produit à étudier par voie intrapéritonéale ou "per os ", Is à 60 minutes avant irradiation $\mathrm{X}$ ou $\gamma$ et à compter le nombre d'animaux survivants le $30^{\mathrm{e}}$ jour suivant l'exposition. Ce test est établi comparativement avec un lot d'animaux témoins.

Les doses d'irradiation les plus utilisées par les radiobiologistes sont la DL $50 / 30 j *$, la DL $70 / 30$ et la DL 100/30j.

La comparaison des résultats radiopharmacologiques obtenus par des auteurs différents est souvent très difficile, même lorsqu'ils emploient un test identique, tel le taux de survie par exemple.

En effet, comme il n'existe jusqu'à présent aucune normalisation, il y a une absence d'unité dans le choix des animaux et en particulier en ce qui concerne la souche, l'âge et le sexe.

Par ailleurs, la différence dans les conditions de vie en animalerie (régime alimentaire, température, nombre d'animaux par cage), la radiosensibilité particulière des différentes souches animales et les modalités différentes d'irradiation (dose et débit variables, dose unique ou fractionnée, rayonnement et temps d'exposition différents, etc.), la pratique des expériences pendant les quatre saisons constituent autant de facteurs compliquant l'interprétation des résultats.

Ainsi, Trne rapporte que, pour une même souche de souris telle la Swiss, les $\mathrm{DL}$ roo/30j varient suivant les auteurs de 600 à $900 \mathrm{R}$ [6r].

On conçoit aisément dès lors que les conclusions rapportées dans la littérature ne soient pas toujours concordantes et on entrevoit tout l'intérêt que présenterait une normalisation des techniques pour évaluer l'effet radioprotecteur.

Il est évident que les résultats en radioprotection, observés chez la souris devront être confirmés par l'expérimentation chez les grands animaux (chien, singe, etc.).

\section{COMPOSÉS RADIOPROTECTEURS}

Devant l'abondance des résultats observés avec des produits de structures très différentes, certains chercheurs ont établi des catalogues de radioprotecteurs [87 à 93]. Des études bibliographiques sur l'ensemble des radioprotecteurs ont fait également l'objet de livres et de nombreuses publications [38,67, 94 à 98].

Nous essaierons de passer en revue, d'une façon très succincte, les substances

* DL $50 / 30 j$ : dose létale pour $50 \%$ des animaux au cours des 30 jours après irradiation.

vOL. $7-\mathrm{N}^{\circ} 4$ 
examinées en radioprotection et nous discuterons l'efficacité des structures dans les séries les plus importantes.

\section{Composés oxyGÉNÉs}

Quelques alcools et en particulier des polyols [75, 99, ror à 104] ont montré une radioprotection modérée chez la souris.

\section{CoMposés AZOTÉS}

Il faut citer en particulier les amines biologiques et tout spécialement la sérotonine qui est un antioxydant physiologique ayant fait l'objet de très nombreuses études $[62,87$, ros à I I 2$]$. Parmi les alcaloüdes nous signalerons la réserpine [ I I 3 à I I s s].

De nombreux composés de synthèse se sont révélés également actifs. Mentionnons : les diamines présentant l'enchaînement N-C-C-N [92, 106, II6 à I18], quelques nitriles [87, 1 19] et quelques composés hétérocycliques [ [ 20 à I 26].

\section{COMposÉS SULFurÉS}

L'un des composés les plus simples, le diméthylsulfoxyde (DMSO) permet une protection de $70 \%$ chez la souris irradiée à une dose létale de rayons X [ 127$]$.

Dans la série des dithiols, le dithio-2,3 propanol (BAL), le dithio-2,3 propanesulfonate de sodium (unithiol) et l'acide dithio-2,3 propionique ont révélé une activité prononcée [ $128, \mathrm{r} 29]$. L'unithiol, plus soluble et moins toxique que le $\mathrm{BAL}$, présenterait une action protectrice qui se maintiendrait pendant une durée considérable [130, I3 $\mathrm{I}]$.

Les dithiolannes ont révélé également un effet protecteur significatif [ $\mathrm{I}_{3} 2$ 2].

\section{CoMposÉs SUlFurÉS ET AZOTÉS}

\section{a) AMINothiols et DÉRIVÉS}

Depuis la découverte de la cystéamine [13], les chercheurs ont respecté la structure $\mathrm{S}-(\mathrm{C})_{n}-\mathrm{N}$ ( $n$ étant égal à 2 ou 3 ) pour atteindre des composés efficaces et ils se sont très vite rendu compte que ces composés devaient présenter dans leur molécule :

- un groupement basique : amine, guanidine ou amidine [67]

Il faut souligner ici que la substitution d'un ou de deux atomes d'hydrogène de la fonction amine par un ou deux radicaux alkylés $[73,136,137]$ ou par un ou deux noyaux aromatiques [ $\left.\mathrm{I}_{3} 6, \mathrm{I}_{3} 7\right]$ ainsi que l'introduction de l'atome d'azote dans un groupement hydrazine $\left[\mathrm{I}_{3} 8, \mathrm{I}_{3} 9\right]$ ou un hétérocycle telle la pipéridine ou la morpholine [ ${ }_{3} 6$ ], diminue l'effet protecteur [140].

Parmi les exceptions à cette règle citons le phénéthyl-N amino-2 éthanethiol possédant une bonne activité [ [ 140 à $\mathrm{I} 43$ ] et $\{($ indolyl-3)-2 éthyl-I' $\}-\mathrm{N}$ amino-2 
éthanethiol-I [ 144] dans lequel il y a une association des structures de la tryptamine et de la cystéamine.

- une fonction thiol libre ou un groupement pouvant être transformé en thiol libre « in vivo ».

Ainsi les dérivés acylés possédant le soufre inclus dans les acides thiosulfurique [145, 146], phosphorothioique [147] et trithiocarbonique [148] sont de bons radioprotecteurs car ils sont susceptibles de libérer très vite "in vivo » la fonction thiol [67]. Ces composés possèdent une structure de "zwitterion ».

Il en est de même de l'amino-2 éthyl-S isothiourée (sous forme de sel) (AET) dont l'activité après injection à l'animal serait liée à une transposition intramoléculaire en (thio-2 éthyl-)guanidine, comme cela se produit en milieu alca$\operatorname{lin}[139]$.

Le remplacement de l'atome d'hydrogène de la fonction thiol dans les composés présentant l'enchaînement S-C-C-N par un radical alkyle, aryle ou benzyle diminue ou annule l'activité radioprotectrice $[26,75]$.

\section{- un chaînon étbylène ou propylène entre les deux bétéro-atomes :}

L'allongement de la chaîne polyméthylénique au-delà de trois carbones intermédiaires entre le soufre et l'azote diminue l'effet radioprotecteur.

Dans le cas des homologues supérieurs de l'AET, si entre la fonction amine et le groupement isothiourée la chaîne polyméthylénique présente plus de trois atomes de carbone, les composés sont inactifs, car ils ne peuvent plus se transposer pour libérer la fonction thiol.

Le remplacement de l'un des atomes d'hydrogène de la chaîne éthylénique de la cystéamine par un radical alkyle peu encombrant ne modifie pas cependant l'effet radioprotecteur.

Ainsi, le groupement méthyle fixé sur le carbone en $\alpha$ du soufre ou de l'azote maintient l'activité puisque le thio-2 propylamine et l'amino-2 propanethiol protègent à $100 \%$ les souris irradiées à la DL $100[26,137,142,150]$. Le remplacement du méthyle par un radical plus important diminue par contre l'action protectrice [ 26,140$]$. Les dérivés dialkylés sur le carbone en $\alpha$ de la fonction aminée sont encore efficaces. Il s'agit de dérivés dialkylés-2,2 amino-2 éthanethiosulfates et phosphorothioates [1 53 ].

Les molécules dans lesquelles les deux carbones intermédiaires entre le soufre et l'azote font partie d'une chaîne polyméthylénique tel l'amino-2 cyclohexanethiol conservent une légère activité. Il y a d'ailleurs peu de différence dans l'efficacité des deux isomères de configuration cis ou trans.

L'oxydation de la fonction thiol dans les structures du MEA et du MEG (conduisant respectivement aux disulfures : cystamine et guanido-éthyl-disulfure (GED) maintient l'activité radioprotectrice [154, 155]. Le GED plus toxique que le MEG, voit cependant son FRD* s'abaisser.

Une oxydation plus poussée du soufre dans les molécules de MEA et de MEG, entraînant la formation de thiosulfinates $[67$, is s à i 57$]$ et thiosulfonates $[67,158]$, mène à des molécules toujours efficaces.

* FRD, facteur de réduction de dose (ou DRF : dose reduction factor) : c'est le rapport entre les doses d'irradiation d'effet identique, avec et sans emploi de substances radioprotectrices.

vOL. $7-\mathrm{N}^{\circ} 4$ 
b) DÉrivés du thiazole, DE LA THIAZOline ET DE LA THIAZOlidine

La découverte des propriétés protectrices du thio-2 benzothiazole [62, 87, I59] a entraîné une série de recherches sur le thiazole, les thiazolines et les thiazolidines.

Parmi les thiazolines, on doit citer l'amino-2 thiazoline [ $\mathrm{r} 60$ ] et la thio-2 thiazoline [ $[\mathrm{I} 6 \mathrm{I}]$, mais c'est avec les dérivés de la thiazolidine que l'on a observé les effets les plus intéressants $[26,146,162,163]$.

En ce qui concerne les aryl-2 thiazolidines tout au moins, l'activité revient à leur édifice propre et non aux produits d'hydrolyse : cystéamine et dérivés carbonylés [164].

\section{c) Acides dithiocarbamiques et Sels}

Dans cette série, le dithiocarbamate d'ammonium, le diméthyl- $\mathrm{N}, \mathrm{N}$ dithiocarbamate de sodium et le diéthyl-N,N dithiocarbamate de sodium protègent pratiquement à $100 \%$, les souris irradiées à la DL 100 [165].

d) ( $\omega$-AMINO ALKYLAMINE ALKYL)-S PHOSPHOROTHIOATES [I 4, Is]

Ces composés répondent aux structures suivantes :

$$
\mathrm{H}_{2} \mathrm{~N}-\left(\mathrm{CH}_{2}\right)_{n^{\prime}}-\mathrm{NH}\left(\mathrm{CH}_{2}\right)_{n}-\mathrm{S}-\mathrm{PO}_{2} \mathrm{H}_{2}
$$

avec $n^{\prime}$ égal à 2, 3, 4, 5 ou 6 chaînons méthyléniques et $n$ à 2 ou 3 méthylènes.

Ces substances ont un effet radioprotecteur considérable avec des FRD pouvant atteindre 2,8 .

De nombreux autres dérivés sulfurés ont également donné des effets radioprotecteurs intéressants.

\section{COMPOSÉS SÉLÉNIÉS}

Le sélénium colloïdal paraît être un bon radioprotecteur [166].

L'action protectrice des amino-acides séléniés paraît être plus grande que celles des homologues sulfurés. Ce serait le cas, notamment de la sélénocystéine, de la sélénométhionine et de la sélénocystine [166].

D’autres composés séléniés se sont avérés également efficaces, en particulier, le sélénoxanthène, la sélénoxanthone et le sélénochrome [r66].

La plus grande efficacité des composés séléniés s'expliquerait par leur aptitude à capter plus rapidement les radicaux libres que les composés sulfurés [ $\left.{ }^{1} 6_{7}\right]$.

\section{MACROMOLÉCULES RADIOPROTECTRICES}

Quelques macromolécules naturelles comme la vasopressine [168], l'ACTH [ 169 ], la mellitine du venin d'abeille [ $\mathrm{I} 70$ ] possèdent une action radioprotectrice intéressante.

De même de nombreuses macromolécules synthétiques et en particulier certains polymères sulfurés $[171,172]$ ont montré une grande efficacité. 
Il s'agit, pour ces derniers, de macromolécules qui, dans la plupart des cas, se scindent par hydrolyse ou action enzymatique dans l'organisme en donnant soit des petites molécules radioprotectrices telles la cystamine, la cystéine, soit des composés micromoléculaires et macromoléculaires conjointement actifs.

Toutes ces macromolécules sulfurées assureraient une action prolongée car elles s'éliminent lentement de l'organisme.

Par ailleurs, leurs structures enchevêtrées leur donnent une haute possibilité d'absorption et de piégeage des radicaux libres.

Les résultats publiés par les auteurs étant bien souvent difficilement comparables, pour les raisons précédemment citées, nous avons essayé d'évaluer par des notes allant de o à 3 l'effet radioprotecteur des diverses substances.

Ces chiffres ne sont pas rigoureux, mais nous ne pouvons pas faire mieux tant que les produits ne seront pas étudiés avec des tests normalisés et en particulier avec un nombre d'animaux statistiquement valable.

Nous proposons d'attribuer pour les taux de survie constatés à la DL 99, les notes suivantes :

- note 3 correspondant à 70 à $100 \%$ de survie;

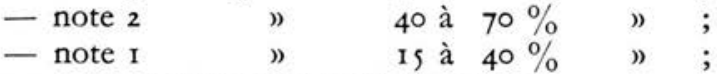

- note o inférieur à is \% de survie.

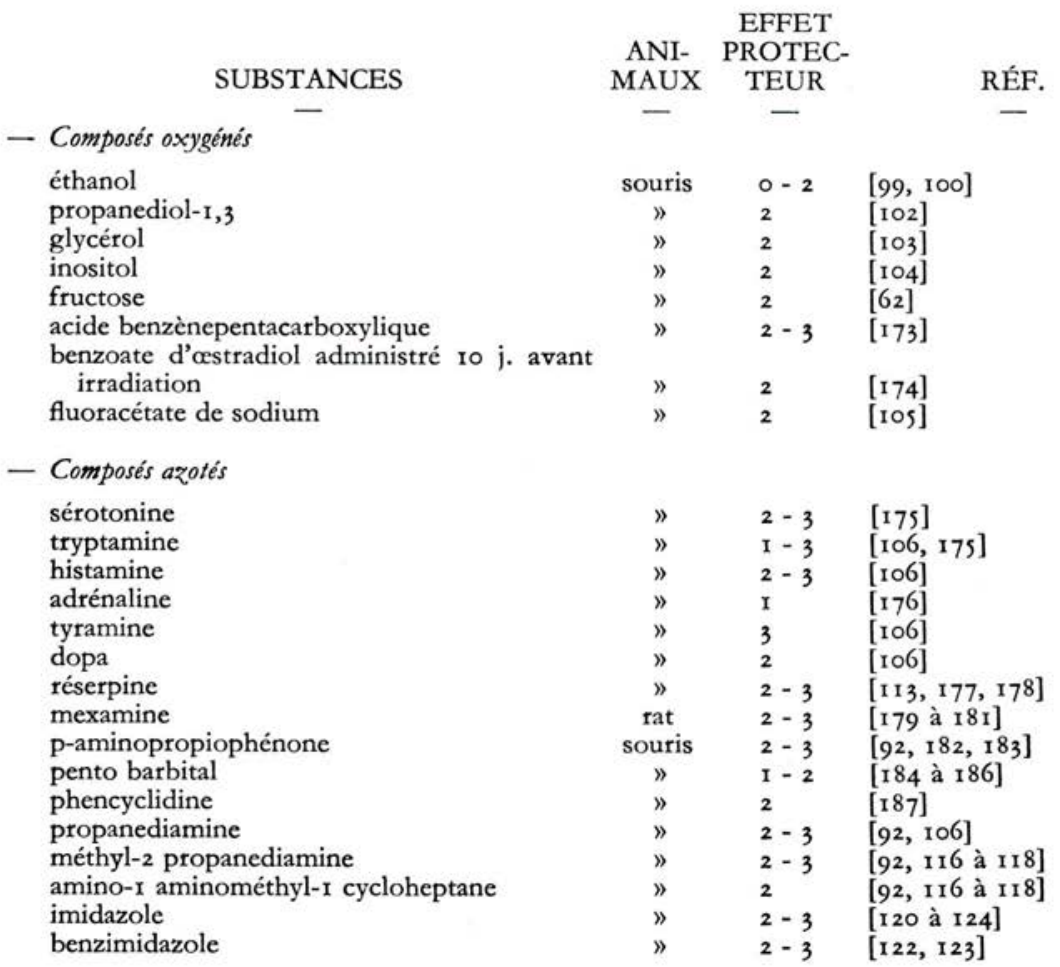

VOL. $7-\mathrm{N}^{\circ} 4$ 


\section{SUBSTANCES}

quinoxaline di $\mathrm{N}$-oxyde

hydroxyacétonitrile

azacytidine et autres analogues

dérivés des bases pyrimidiniques

\section{- Substances sulfurées}

diméthylsulfoxyde

dithio-2,3 propanol

dithio-2,3 propanesulfonate de sodium

acide dithio-2,3 propionique

dithiolannes

\begin{tabular}{|c|c|c|}
\hline ANI- & $\begin{array}{l}\text { EFFET } \\
\text { PROTEC- }\end{array}$ & \\
\hline MAUX & TEUR & RÉF. \\
\hline - & - & - \\
\hline$"$ & $2-3$ & {$[188,189]$} \\
\hline$»$ & $1-3$ & [87] \\
\hline$"$ & & [9] \\
\hline$"$ & 2 & {$[190,19 \mathrm{r}]$} \\
\hline souris & $2-3$ & {$[127,192$ à 195$]$} \\
\hline$"$ & $x=2$ & {$\left[87,128,1_{30}, 1_{33}, 1_{96}\right]$} \\
\hline$"$ & 2 & {$[128,130,13 \mathrm{I}]$} \\
\hline " & $2-3$ & [1 28] \\
\hline$"$ & $1-2$ & [132] \\
\hline
\end{tabular}

\section{- Dérivés du thiazole, de la tbiazoline et de la tbiazolidine}

thio-2 benzothiazole amino-2 thiazoline thiazolidine alkyl-2 thiazolidines aryl-2 thiazolidines

$\begin{array}{cll}\text { souris } & 3 & {[87,204]} \\ " & 2 & {[205]} \\ " & 0-2 & {[26,87,162]} \\ " & 1-3 & {[26,162]} \\ " & 1-3 & {[26,162]}\end{array}$

[I3]

[I 33

[133]

[197]

[197]

[143]

[134]

[198]

$[36,144]$

[134]

[145]

[147]

[153]

[i 199$]$

[147]

[148]

[I 34]

[134]

$[75,140,142,200]$

[140, 142]

$[140,142,16 \mathrm{I}]$

$[75,201]$

[202]

[26]

[26]

[26]

[26]

[203]

[13]

[I 54 ] 


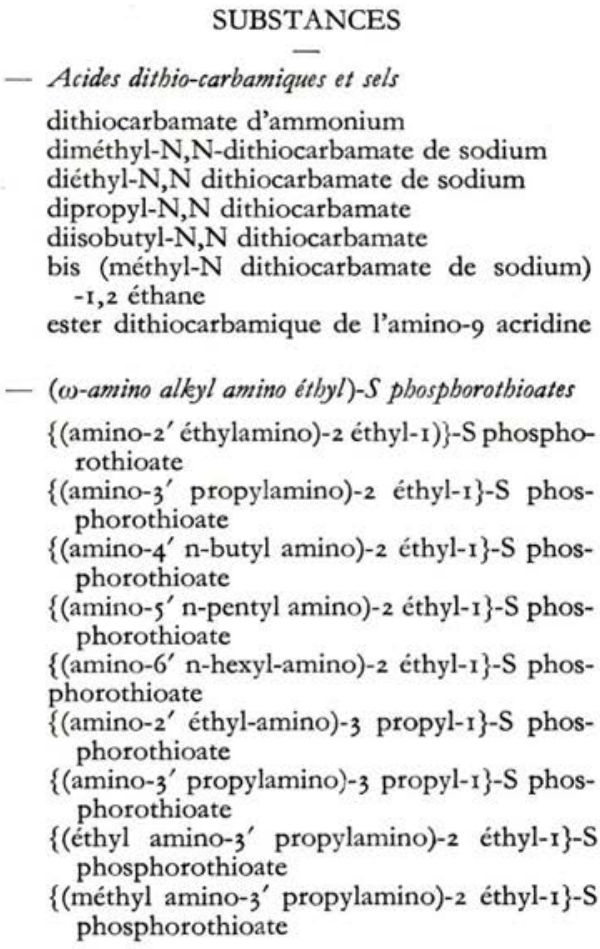

\begin{tabular}{|c|c|c|}
\hline souris & 3 & [I4] \\
\hline$»$ & 3 & [14] \\
\hline$"$ & 3 & [14] \\
\hline$"$ & 3 & [I4] \\
\hline$"$ & 3 & [14] \\
\hline$»$ & 3 & [14] \\
\hline$»$ & 3 & [30] \\
\hline$»$ & 3 & [15] \\
\hline souris & 3 & [Is] \\
\hline
\end{tabular}

\begin{tabular}{|c|c|c|c|}
\hline $\begin{array}{l}\text { ANI- } \\
\text { MAUX }\end{array}$ & $\begin{array}{c}\text { EFFET } \\
\text { PROTEC- } \\
\text { TEUR }\end{array}$ & & RÉF. \\
\hline - & - & & - \\
\hline " & 3 & {$[165]$} & \\
\hline " & 3 & {$[165]$} & \\
\hline 》 & 3 & [165] & \\
\hline 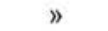 & 2 & {$[26]$} & \\
\hline 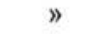 & 2 & {$[26]$} & \\
\hline 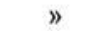 & 3 & {$[26]$} & \\
\hline ” & 3 & [206] & \\
\hline
\end{tabular}

\section{AUTRES DÉRIVÉS SULFURÉS}

(thio-2 éthylamino)-4 propionamide (amino-3 propyl-1)-N amino-2 éthanethiol bis (thio-2' éthyl-N, $N^{\prime}$ ) propanediamine- 1,3 bis [(acide phosphorothioïque-S)-2 éthyl-N,N'] -propanediamine- I, 3

$\begin{array}{cll}\text { souris } & 3 & {[207,208]} \\ n & 3 & {[209]} \\ " & 3 & {[210]} \\ n & 3 & {[15 \text { bis }]}\end{array}$

\section{ASSOCIATION DE RADIOPROTECTEURS}

Maisin et Mattelin ont montré que le FRD qui est de 1,7 pour l'AET, passe à 3,6 avec le cocktail formé par l'AET, le MEA, le glutathion, la sérotonine, la cystéine et la moelle osseuse [2 11 I]

WANG et HASEGAWA, étudiant la protection obtenue avec l'association formée par la sérotonine, l'AET et le MEA contre des doses supralétales de rayonnement $\mathrm{X}$, constatent que la DL so obtenue est de $\mathrm{r} 850 \mathrm{R}$ avec le mélange au lieu de $850 \mathrm{R}$ chez les témoins non protégés [ 212 à 214 ].

Ces auteurs ont essayé la même association de radioprotecteurs sur les souris irradiées à 800 , I roo et $\mathrm{r} 400 \mathrm{R}$ pendant cinq fois successives avec un intervalle vOL. $7-\mathrm{N}^{0} 4$ 
de 28 jours entre chaque irradiation. Les pourcentages de mortalité observés après la cinquième irradiation sont de 55, 95 et roo \% pour les doses de radiations totales accumulées de 4000 , s 500 et 7000 röntgens.

Cela montre de façon claire l'importance de telles associations dans la protection contre des doses répétées qui sont chacune supralétales (dans cette expérience la DL 100 du groupe contrôle était de 800 röntgens [21 5 ].

Beaucoup d'autres synergies ont été constatées, nous citerons les principales associations étudiées jusqu'à présent :

- cystamine, streptomycine [215];

- AET, cystéine, glutathion, sérotonine [217];

- cystéine, polyoside extrait d'Eberthella typhosa [218];

- L. cystéine, N-acétyl - DL - homocystéinethiolactone, acide folique, ferrosulfate, D-fructose, vitamines $\mathrm{B}_{1} 2$ et $\mathrm{C}[2 \mathrm{r} 9]$;

- substances soufrées, vitamines E (cité dans [37]);

- insuline, glucose, vitamine $\mathrm{K}$ (cité dans [37]);

— insuline, glucose, méthandrosténolone (cité dans [37]);

- sérotonine, adénosine monophosphate [220, 221]);

- sérotonine, vitamine B 6 (cité dans [37]);

- acide $\gamma$-hydroxybutyrique, vitamine B 6 (cité dans [37]);

- acide $\gamma$-hydroxybutyrique, ACTH, chlorpromazine (cité dans [37]);

- acide $\varepsilon$-amino caproïque, acide p-aminométhylbenzoïque (cité dans [37]);

- aminoacides, vitamines [222];

- corticoïdes, antibiotiques, vitamines [223].

\section{CONCLUSION}

Bien que des progrès importants en radioprotection aient été réalisés, les recherches dans ce domaine doivent être pousuivies et encouragées, car elles sont d'un intérêt considérable.

Le développement des études sur les mécanismes permettra peut-être de mettre en évidence, pour quelques séries de radioprotecteurs, la présence de sites récepteurs spécifiques, de configuration bien déterminée, au niveau des protéines.

En effet, notre attention a été attirée par des observations d'ordre structural. Ainsi H. IrIe a montré que l'amino-2 cyclohexanethiol (ACHT) [203] était moins actif que l'amino-2 éthanethiol et que, par ailleurs, les deux isomères cis et trans de l'ACHT présentaient une légère différence d'activité.

L'isomérie optique semblerait jouer également un rôle, le bromure de D-amino-2 butyl isothiouronium étant deux fois plus actif que l'isomère L [224].

En radioprotection ainsi que sur le système nerveux, les effets de certaines molécules telle la chlorpromazine, dont le comportement a été comparé à un semi-conducteur, font apparaître un point commun entre les molécules appartenant à ces deux groupes et entrevoir la naissance d'une pharmacologie électronique (cité dans [37]).

La notion de radioimmunisation [225] qui se développera aussi dans les années à venir offre des perspectives nouvelles qui viennent s'ajouter aux précédentes. 
Enfin, les physiciens pourront peut-être contribuer à l'avancement des recherches en éclairant les chimistes sur les phénomènes physiques initiaux, notamment sur les variations possibles du spin des électrons périphériques des atomes, qui se produisent sous l'action du rayonnement ou sous l'action de l'oxygène, par exemple.

L'élucidation de ces processus devrait permettre d'envisager une synthèse plus orientée de substances nouvelles. Il faudrait poursuivre les recherches, vers l'obtention de substances présentant un effet préventif. On peut envisager plusieurs possibilités.

I - Action préventive à long terme. La mise au point de nouveaux « vaccins » pourrait être également poursuivie.

En effet, il est possible que des substances plus efficaces que l'uréase (produite par la fève Jack [226] ou que des cultures de Salmonella [227], qui administrées longtemps à l'avance, ont des propriétés immunisantes chez la souris contre les radiations, puissent encore être découvertes.

2 - Action préventive à moyen terme. Déjà des molécules comme la méthandrosténolone (cité dans [37]), anabolisant protéique, administrée per os, 7 jours avant l'exposition aux rayonnements, a montré un effet protecteur.

3 - Action préventive à court terme. La recherche de molécules efficaces par administration juste avant irradiation doit également s'amplifier.

En fait, le radioprotecteur idéal devra posséder une action protectrice importante pendant une longue durée et présenter en outre une toxicité réduite, ainsi qu'une absence d'effets cumulatifs.

Il serait souhaitable que la synthèse de nouveaux radioprotecteurs soit dirigée vers des substances capables d'inhiber les phénomènes physiques initiaux qui se produisent dans les $10^{-13}$ à $10^{-9}$ seconde suivant le transfert d'énergie.

Nous pensons que, parmi ces substances, les donneurs d'hydrogène atomique et les donneurs d'électrons se trouveront en bonne place, ces derniers se situant parmi les molécules dont le potentiel d'ionisation est le plus bas.

Ces substances devront aussi être capables de faire retomber à leur niveau énergétique de base les biomolécules excitées par le rayonnement.

Des activateurs métaboliques capables de stimuler la voie oxydative directe au niveau des enzymes ainsi que des inducteurs de la catalase doivent être recherchés.

On connaît en effet toute l'importance de cette dernière enzyme dans la décomposition de l'eau oxygénée formée dans le métabolisme oxydatif cellulaire normal.

Par ailleurs, il faut réaliser un examen systématique d'association de radioprotecteurs, car il a été démontré que plusieurs radioprotecteurs administrés ensemble peuvent augmenter considérablement l'efficacité.

Pour conclure, souhaitons que la science arrive à mettre à la disposition de l'homme, dans un avenir proche, des moyens de lutte efficaces, contre les radiations qui ne seront utilisées qu'en temps de paix pour le servir.

S'il était nécessaire, formulons des vœux pour que l'histoire récente le rappelle à la sagesse et lui évite d'employer les radioprotecteurs lors des lueurs d'Apocalypse.

vol. 7 - No 4 


\section{BIBLIOGRAPHIE}

[I] Griffith W.H., Dyer H.M. Life Sciences Research office. Federation of american Societes for experimental biology, Washington D.C. is janvier 1966.

[2] Kayser P. Bull. Soc. Pharm. Nancy, 62 (1964), 7-17.

[3] Mechalr D. Effets biologiques des rayonnements ionisants. In : Utilisations de l'énergie mucléaire, ed. Masson et Cie, Paris, quinzaine des Sciences nucléaires, Montpellier (24 mars 8 avril 1962$), 263-272$.

[4] Georges J. Actualités pharmaceutiques (1964), 307-316.

[5] Genaud P. Bull. Fédération franfaise des Amicales de Pharmaciens de réserve, 3 (1959), 31 2-335.

[6] Cristau B. Pharmacia Meditteranea, Cronoca delle giornate Congressuali, Ferrara-Venezia (1964), 88-117.

[7] LORENZ E. In : Zirkle biological effects of external and gamma radiation (McGraw-Hill), cité dans [s].

[8] Lorenz E., Congdon C.C. Radio-activity Biol. effects of ionising radiations. Ann. Rev. Med., 5 (1954), 323-38.

[9] Latarjet R., Ephrati E. C.R. Soc. Biol., $\mathbf{I}_{42}$ (1948), 497.

[10] Hervé A., BACQ Z.M. C.R. Soc. Biol., 143 (1949), 881.

[ir] Patr H.M., Tyree E.B., Straube R.L., Smith D.E. Science, iro (1949), 2 I 3.

[12] Mole R.H., Philpot J. St. L., Modges C.R.V. Nature, 166 (1950), 5 I5.

[13] Bace Z.M., Hervé A., Fischer P., Lecomte J., Blavier J., Dechamps G., Le Bihan H., RAYET P. Arch. Intern. Physiol., 59 (1951), 442.

[14] Piper J.R., Stringfellow C.R. Jr., Elliott R.D., Johnston T.P. J. Med. Chem., i 2 (1969), $236-243$.

[is] Piper J.R., Stringfellow C.R. Jr., Johnston T.P. J. med. cbem., 12 (1969), 244-253.

[is bis] J. Med. cbem., 9 (1966), 563.

[16] BACQ Z.M. Triangle, $\mathrm{n}^{\circ}$ I (1961), i-1 r.

[i7] Hollaender A., Stapelton G. Physiol. Rev., 33 (1953), 77.

[18] Nizet A., Bace Z.M., Hervé A. Arch. Intern. Physiol., 6o (1952), 448.

[ig] Sanner T., Pihl A. Scand. J. Clin. Invest., 22, suppl. 106 (1968), 53.

[20] Sanner T., Pihl A. Radiat. Res., 37 (1969), 216.

[21] Alexander P., Dean C.J., Lehman A.R., Armerod M.G., Feldschreiber P., Serianni R.W. Deuxième symposium inter. sur les radiosensibilisants et les radioprotecteurs. Radiation protection and sensitization, Rome (1969), 15-34, ed. H.I. Moroson et M. Quintiliani.

[22] PIHL A., SANNER T. Radiation protection and sensitization, Rome (1969), 43-55.

[23] Bavq. Bull. Académie Royale de Médecine, 2, VI (1966), II5-14I.

[24] BACQ Z.M. La protection chimique contre les radiations ionisantes chez les mammifères. Conférence donnée le 16 janvier 1967 à l'Institut de Pharmacie Industrielle de Montpellier.

[25] Tarasenko A.G., Graevskij E.J.A., Konstantinova M.M., Mandrugin A.A., Nekrasova I.V., Fedosegv V.M. Dokl. Akad. Nauk S.S.S.R., r87, nº I (1969), 203-206.

[26] Yu Ratchinskiy F., Mozjukin A.S., Slavatchevskaia N.M., Tank L.I. Usp. Kbim. (Progrès de la chimie), Moscou, 28, $\mathrm{n}^{\circ}{ }_{12}$ (1959), I488-1522.

[27] Bace Z.M., Alexander P. Radiobiology Symposium, Londres, I955.

[28] Latarjet R., Eckert B., Demerseman P. Radiat. Res. (1963), suppl. 3, 247.

[29] Pihl A., Eldjarn L. Pharmacol. Rev., ro (1958), 437.

[30] Eldjarn L., Pihl A. Progress in Radiobiology, Edimbourg, 1956.

[31] Eldjarn L., PrhL A. Mechanism of protective and sensitizing action in mecbanisms in radiobiology. Ed. M. Errera et A. Forsberg, New York, Academic Press, 2, (1960), 231.

[32] EldJARN L. Cbemical protection against ionising radiation, Radiocbemistry or cellular pbysiology dans Strablenvirkung und milieu, p. 232. Ed. Fritz Niggli, Munchen, Urban et Schwartzenberg (1962).

[33] Jellum E. Int. J. Radiat. Biol., ro (1966), 577.

[34] Kollmann G., Shapiro B., Martin D. Radiat. Res., 31 (1967), 721.

[35] Danysz A. Act. Pharmacologiques, 22 (1969), 233-259.

[36] Bergmann E.D. Mécanismes de l'action biologique des rayonnements ionisants, radioprotection chimique; conférence présentée à la Direction des Recherches et Moyens d'Essais, 5 , bis avenue de la Porte de Sèvres, Paris $\left(15^{\mathrm{e}}\right), 18$ novembre 1969. 
[37] Perrt. La radiation interne. Le pbarmacien de Réserve, no 4 (1970), $461-517$.

[38] BACQ Z.M. Chemical protection against ionising radiation, éd. Charles Thomas, Springfield (Illinois) (1965), U.S.A.

[39] BAcQ Z.M., Goutier R. Brookhaven Symposium Biol., 20 (1967), 241.

[40] Eldjarn L., Jellum E. In : Radiation Damage to the biological molecular information system with special regard to the role of $\mathrm{SH}$ groups. Vienna : International-Atomic Energy Agency (1969), 45 .

[41] BACQ Z.M., Alexander P. Nature, 203 (1964), 162.

[42] Revesz L., Modig H. Nature, Londres, 207 (1965), 430.

[43] Modig H. ,Revesz L. Int. J. Radiat. Biol., 13 (1967), 469.

[44] Modig H., Revesz L. Arzneim-Forsch., 18 (1968), 1 I 56.

[45] KruUv J., Sinclair W.K. Radiat. Res., 36 (1968), 45.

[46] Sinclair W.K. Radiat. Res., 33 (1968), 620.

[47] Sinclair W.K. Science, 159 (1958), 442.

[48] Elkind M.M., Sinclair W.K. In : Current topics in Radiation Research, I (1965), 165 (Amsterdam : North Holland Publishing Co.).

[49] Painetr R.B., Cleaver J.E. Nature, Londres, $2 x 6$ (1967), 369.

[so] Goutier R., BAugnet-MAHIEU L. In : Radiation damage to the biological molecular information system witb special regard to the role of $\mathrm{SH}$ groups. Vienna, International Atomic Energy Agency (1969).

[5i] Goutier R., Baugnet-Mahieu L., Berg T.L. Bull. de l'Académie Royale de médecine de Belgique, VIIe série, 7 (1967), 796.

[s2] Danysz A., Kocmierska-Grodzka D., Killar M., Kruszewska J., Panek R. Biul. W $A M, 7$ (1965), I4I-I 44 .

[53] Danysz A., Kocmierska-Grodzka D., Niewiarowski S., Poplawski A., Worowski K., REJNIAK K. Biul. WAM, 7 (1965), 65-70.

[54] Danysz A., Kocmierska-Grodzka D., Niewiarowski S., Wisniewski K., Prokowicz J. Folia Hoaematologica, 87, r (1967), 128-134.

[5s] Danysz A., Kocmierska-Grodzka D., Panek R., Mackowiak J. Med. Radiol, 2 (i968), 3-9.

[56] Danysz A. Nukleonika (cité dans [35]).

[57] Danysz A., Kocmierska-Grodzka D., Niewiarowski S., Prokopowicz J., Woroski K., REJNIAK K. Sbornik vedeckych praci lekarske Fakulty Ku, v Hradci Kralove, 9 (1966), 4-5.

[58] Kocmierska-Grodzka D., Danysz A. Acta Pbysiol. Pol., 19 (i968), i-5.

[59] Schubert J. Cbemical Engeneering News, 39, n० 14 (196r), $25-27$.

[60] Schubert J. Abstracts 139th National Meeting. American cbemical Society, 9 N (1961).

[6I] TrNe J. Contribution à l'étude de l'activité radioprotectrice éventuelle de quelques métaux à l'état ionique ou chelate. Tbèse de l'Université de Lyon, Section de Pbarmacie, 1963 .

[62] Bace Z.M., Alexander P. Principes de radiobiologie, Sciences et Lettres, Liège; Masson et Cie, Paris, 1955. In : Fundamentals of Radiobiology, Ire éd. : Butterworth, Londres, 1955; ${ }_{2}$ e éd. : Pergamon Press, Oxford, 196r.

[63] Rrnaldi R. La protection cbimique contre les effets des radiations ionisantes. Quinzaine des Sciences Nucléaires, Montpellier (24 mars - 8 avril 1962), 183-196. Ed. Masson et Cie.

[64] Lacassagne (cité dans [63]).

[65] Dowdy, Bennetr, Chastain (cité dans [63]).

[66] Markovich $\mathrm{H}$. Etude radiobiologique du système lysogène d'Escbericbia coli $\mathrm{K}$ I2, Tbèse de l'Université de Paris, 1957.

[67] FoYe W.O. J. Pbarmaceutical Sciences, 58 , $\mathrm{n}^{\circ} 3$ (1969), 283-300.

[68] Plzak V., Doull. J. Univ. Chicago, USAF, Rad. Lab. Quart. Rep., 32 (1959), 68.

[69] Salerno P.R., Friedell H.L. Radiat. Res., I (1954), 559.

[70] Goepp R.A., Fitch F.W., Doull J. Radiat. Res., 31 (1967), 149.

[71] Sonka. Voie des pentoses. Univ. Charles, Faculté Médecine, Prague, no 5, 1966 (cité dans [37]).

[72] Patr H.M. Physiol. Rev., 33 (1953), 35.

[73] Hagen U. Advances in radiobiology, Londres (1957), 187.

[74] Cier A., Nofre C., Ecalle R. C.R. Acad. Sci., 255 (1962), $2840-2842$.

[75] Alexander P., Bace Z.M., Cousens S.F., Fox M., Herve A., Lasar J. Radiat. Res., 2 (1955), 392.

[76] Alexander P., Fox M. J. Chim. Pbys., 50 (1953), 415.

[77] Duboulcz P., Dumas J., Vigne J. C.R. Soc. Biol., r44 (1950), 1080.

vOL. $7-\mathrm{N}^{\circ} 4$ 
[78] Horgan V., Philpot J. Brit. J. Radiol., 27 (1954), 63.

[79] Zhuravlev K.I. Problèmes de la nutrition, r6, 4 (1958), 60.

[80] Zhuravlev K.I. Bjul. Radiatsionnoi Medics, 2 (1957), 72.

[81] Honnan R., Boag J. Nature, 169 (1952), is 2.

[82] Yu Ratchinskiy F., Slawatchevskaia N.M. Rapport présenté à la se Session scientifique sur la chimie des composés organiques soufrés et azotés contenus dans les pétroles et leurs dérivés (UFA) (1959), 7x (cité dans [26]).

[83] Cheymol J., Deyson G., Adolphe M., Hertz F. Tbérapie (1964), ro59-1070.

[84] Trabert M., Maessen Van Der. C.R. Soc. Biol., I51 (1957), 1624-1626.

[85] Whitfield J.F., Rixon R.H., Youdale T. Exp. Cell. Res., 27 (1962), I43-147.

[86] Whitrield J.F., Rixon R.H. Exp. Cell. Res., 27 (1962), is4-is7.

[87] Doull J., PlzaK V., BroIs S.J. A survey of compounds for radiation protection school of aerospace Medicine US AF. Aerospace Medical Division (AFSC). Brooks Air Force Base, Texas (1962).

[88] Trunov L.A., VAsIL'EV G.A., PARIBOK V.P. Radiation protection bandbook, traduit de la publication de l'Academy of Sciences USSR, Moscou, Leningrad, 196r, United States Atomic Energy Commission. Division of Technical information.

[89] Huber R., SPODE E. Biologisch-chemischer Strablenscbutz, Akademie-Verlag, Berlin (2 vol.) $(1963)$.

[90] Huber R., Spode E. Biologisch-chemischer Strablenscbutz, Akademie-Verlag, Berlin (2 vol.) $(1963)$.

[9i] Sablayrolles C., RobBe Y. Effets radioprotecteurs de substances minérales et organiques, vol. I et II (1965), éd. Direction des recherches et Moyens d'Essais, 5 bis, av. de la Porte de Sèvres, Paris.

[92] Roвbe Y., RAndon M. Effets radioprotecteurs de substances minérales et organiques, vol. III (1966); vol. IV (1967); vol. V (1968); éd. : idem.

[93] Robbe Y., Randon M., Valentin M., Chevron F., Fernandez J.P. Effets radioprotecteurs de substances minérales et organiques. Index, 1969. Ed. : idem.

[94] "Annual Reports in Medicinal Chemistry, 1965, 1966, 1967". C.K. Cain Ed. Academic Press, New York, N.Y. (1966-1968).

[95] "Progress in Biochemical Pharmacology », vol. 1, R. Paoletti et R. Vertua, eds : Butterworths, Washington, D.C. 1965 (cité dans [67]).

[96] Melching J., Streffer C. Prog. Drug. Res., 9 (1966), ir.

[97] Thомson J.F. Radiation Protection in Mammals, Reinhold, New York, N.Y. 1962.

[98] Atrinson E.R. In : Antiradiation drugs-A. Bibliography. Arthur D. Little, Cambridge, Mass (cité dans [67]).

[99] Paterson E., Matrhews J.J. Nature, x68 (i95 r), ir 26.

[100] Luchnik N.V. Atomnaya energiya, 5 (1956), 134-135.

[ror] Plzak V., Root M., Doull J., USAF. Radiat. Lab. Quarterly Progress Report Univ. Chicago, 42 (15/r/1962).

[102] Mozjukhin A.S., Yu Ratchinski F., Tank L.I. Med. Radiologiya, 5, 4 (i960), 78-81.

[103] Bace Z.M., Herve A., Fischer P. Bull. Acad. Roy. Med. Belg., 18 (1953), 226.

[104] BrINKAN R. Cellular basis and aetiology of late somatic effects of ionising radiation. A symposium, Ed. R.J.C. Harris, Academic Press, New York, N.Y. (1963), 179.

[ros] Novak L., Sikulova J., Hosek B., Mrsustova J. Radiat. Res., 40, no 2 (1969), 430-9.

[106] Bace Z.M. Acta Radiobiologica, 4I (1954), 47-55.

[107] LangendorfF H., Koch R. Strablentberapie, 102, 2 (1957), 58, 60.

[io8] Barnes J.H., Lowman D.M.R. J. Rad. Biol. G.B., I4 (1968), ñ i, 87-8.

[rog] Vitrorio P.V., Watkins E.A., Dziubaloblehm S. Canad. J. Physiol. Pharmacol., 47, $\mathrm{n}^{\circ} \mathrm{I}$ (1969), 65-71.

[riro] Peter Dukor Von. Ansder, Dermatologischen Universitats Klinik (Vorsteher Pr Dr R. Schuppli). Versuche zum Mechanismus der Strahlenschutzwirkung von oxytryptamin derivaten (cité dans [9r]).

[iir] Gray J.L., Tew J.T., Jensen H. Proc. Soc. Exptl. Biol. Med., 80 (1952), 604.

[i12] LangendorfF H., KоCH R. Strablentberapie, $9^{8}$ (1957), 245.

[ir3] Langendorff M., Melching H., Langendorff H., Koch R., Jacques R. Strablentherapie, 104, 3 (1957), 330-344.

[ir4] Melching H., Langendorff M. Naturwissensch, 44, 13 (1957), 377.

[irs] Langendorff H., Melching H., Ladner H. Strablentherapie, ro8, 2 (1959), 25 I-256.

[II6] Rовве Y. Recherche de radioprotecteurs, Synthèse étude structurale et radiopharmacologie, Thèse de doctorat en pharmacie, Montpellier (1968). 
[ir7] Granger R., Orzalesi H., Robbe Y. Trav. Soc. Pbarm. Montpellier, 24, no 4 (1964), 244-255.

[ir8] Arnaud G., Fatome M., Granger R., Orzalesi H., Robbe Y. Cbimie Tberapeutique, no 2 (1969), I36-145.

[irg] Hernadi F., Nagy Z., Kovacs P., Dezsi Z., Valyi-Nagy T. Acta biol. Acad. Sci. bungar, 19, $\mathrm{n}^{\circ}{ }_{3}$ (1968), $257-67$.

[ 1 20] Rinaldi R., Bernard Y. Centre d'études nucléaires de Grenoble. Commissariat à l'énergie atomique Fr. (1962), Rapport CEA, no 2207.

[121] Mourret A., Rinaldi R. C.E.N., Grenoble, Rapport C.E.A., Fr. (i 968$)$, nº 3010.

[1 22] Pierrotri T., Roushdy H., Polverelli M., Mazza M. C.E.N., Grenoble, Rapp. C.E.A., Fr. (1969), n० 3679 .

[123] Roushdy H., Pierrotri T., Polverelli M., Z. Naturforsch., B. Disch., 24, no s (1969), 622-30.

[124] Polverelli M., Teoule R., C.E.N. Grenoble, Rapp. C.E.A. 3809 (1969).

[r25] Plzak V., Roor M., Doull J., USAF Radiation, Radiat. Lab. Quarterly Progress Report, Univ. Chicago, 53 (15/10/1964).

[126] Plzak V., Root M., Doull J. USAF Radiation, Radiat. Lab. Quarterly Progress Report. Univ. Chicago, 55 (15/4/1965).

[1 27] Krm S.E., Moss Wis. Healtb physics, 13, 6 (1967), 601-606.

[1 28] Graevskiy E.J., Konstantinova M.M. C.R. Acad. Sci., URSS, 136, s (1961), r 219 -1 222.

[129] Schroder E., Magdon E., Semenov L. Strablentberapie, 132, 2 (1967), 306-31.

[130] Ya Arbuzov S., Koporkova B.P. Rapport sur le problème des thiols en médecine, Kiev (1957), 3-4 (cité dans [1 128$]$ ).

[13I] YA Arbuzov S. Les processus de réparation et de compensation dans la maladie des rayons, Moscou, 1959 (cité dans [1 128$]$ ).

[132] Fatome M., Poutrain P., Granger R., Orzalesi H., Robbe Y., Randon M., Valentin M., Chevallet P. Chimie Tbérapeutique, no s (1970), 327-331.

[133] Doherty D.G., Burnetr W.T. Jr., Shapira R. Radiat. Res., 7 (1957), 13-21.

[134] Doherty D.G., Burnetr W.T. Proc. Soc. exp. Biol. (N.Y.), 89 (1955), 31 2-314.

[i35] Doherty D.G., Shapira R.S., Burnetr W.T., J. Amer. Chem. Soc., 79 (1957), 5667.

[136] Langendorff H., Koch R. Strablentberapie, 99 (1956), 567.

[137] Mozjukhin A.S., Yu Ratchinskiy F., Slavatchevskaia N.M., Tank L.I. Thème du rappport à la conférence scientifique VMOLA, S.M. Kirov. Sur : Les problèmes des lésions multiples de la prévention et du traitement de la maladie des rayons, Leningrad (1958), p. 33 (cité dans [26]).

[138] Atrinson E.R., Handrick G.R., Bruni R.J., Granchelli F.E. J. Med. Chem., 8 (i965), 29.

[139] Rose F.L., Walpole Al. In : Progress in Biochemical Pharmacology, vol. I, Butterworths, Washington, D.C. (1965), 432.

[140] TANK L.I. Rapp. présenté à la conférence scientifique de l'Institut ukrainien de recherches de chimie sanitaire sur la question: Les thiols en médecine, Kiev (1957), 83 (cité dans [26]).

[i4i] Belai V.E., Vasiliev P.V., Vedernikov L.N., Gorban G.M., Mozjukhin A.S., Pereponov G.A., Yu Ratchinskin F., Sans'onov P.P., Slavatchevskaya N.M., Tank L.I., Timofeev G.I., Chernenko G.T. Rapport présenté à la conférence scientifique de l'Académie de médecine militaire S.M. Kirov, 27-29 mars 1957, Leningrad (1957), I3 (cité dans [26]).

[142] TANK L.I. Rapport présenté à la conférence scientifique de l'Institut de méd. exp. de l'Acad. des Sciences méd. d'URSS sur la radiobiologie, Leningrad (1957), 50 (cité dans [26]).

[i 43] Ferris A.F., Salerni O.L., Schutz B.A. J. Med. Chem., 9 (i966), 39 I.

[144] Kalir (Kaluszyner) (cité dans [36]).

[145] Holmberg B., Sorbo B. Nature, 183 (1959), 832.

[146] Kaluszyner A., Czerniak P., Bergmann E.D. Radiat. Res., 14 (1961), 23-28.

[147] Hansen B., Sorbo B. Acta Radiol., 56 (196r), i4r.

[148] Foye W.O., Mickles J., Duvall R.N., Marshall J.R. J. Med. Chem., 6 (i963), 509.

[i49] Khym J.X., Shapira R., Doherty D.G. J. Am. Chem. Soc., 79 (1957), s663.

[iso] Ya Arbuzov S. Bull. Ac. Sciences, Méd. URSS, 6 (1958), 10.

[igi] Carroll F.I., While J.D., Wall M.E. J. Org Cbem., 28 (1963), i 240.

[is2] Stacy G.W., Barnetr B.F., Strong P.L. J. Org. Chem., 30 (r965), 592.

[is3] Piper J.R., Stringfellow C.R. Jr., Johnston T.P. J. Med. Cbem., 9 (i966), gi i.

vOL. $7-\mathrm{N}^{\circ} 4$ 
[i54] Schwartz E.E., Shapiro B. Radiat. Res., 13 (1960, 780.

[iss] Shapiro B., Schwartz E.E., Kollman G. Radiat. Res., 18 (1963), 17.

[i56] Klayman D.L., Milne G.W.A. J. Org. Cbem., 31 (1966), 2349.

[is7] Foye W.O., Hebb A.M., Mrckles J. J. Pharm. Sc., 56 (1967), 292.

[is8] Wang R.I.H., Hasegawa A.T. (cité dans [67]).

[r59] Doull J., Plzak V., Brois S., USAF Radiat. Lab. Univ. Chicago, Status Report, $\mathrm{n}^{\circ} 2$ $(\mathrm{I} / 8 / \mathrm{rg} 6 \mathrm{I})$.

[160] Shapira R., Doherty D.G., Burnetr W.T. Jr. Radiat. Res., 7 (1957), 22.

[161] JACoBus D.P., SwEENEY T.R. (cité dans [67]).

[162] Fatome M., Poutrain P., Granger R., Orzalesi H., Robbe Y., Randon M. Chimie Thérapeutique, $\mathrm{n}^{\circ}$ S (1970), 312-317.

[163] Granger R., Orzalesi H., Robbe Y. Trav. Soc. Pbarm., Montpellier, 27, no I (1967), 23-27.

[164] Granger R., Orzalesi H., Robbe Y., Chapat J.P., Terol A., Randon M., Brtoun J., Simon F., Fernandez J.P. Chim. Thérap., no 6 (1971), 439-441.

[165] Bexкum D. Van. Acta Pbysiol., Pbarma. col., Neerl., 4 (1956), 503-523.

[166] Breccia A., Badiello R., Trenta A., Matri M. Radiat. Res., USA, 38, no 3 (1969). 483-92.

[167] Villar-Palasi V. Pbarmacia Mediteranea, 5 (1964), 67.

[168] Gray J., Moulden E., Tew J., Jensen H. Proc. Soc. Exptl. Biol. Med., 79 (1952), 384.

[i69] Rogozkin V.D., Musabekov Dt., Shapiro G.A. Bjull. ekesper. Biol. Med. SSSR, 68, n 9 (1969), 30-2.

[170] Ginsberg N.J., Dauer M., Slotta K.H. Nature, G.B., 220, nº s (1968), 174, 1334.

[171] PANY J. Naturwissenscbaften, 50 (1963), 734.

[172] Ringsdorf H. Strablentberapie, 132, 4 (1967), 627-635.

[173] Barness J.H. Nature, 205 (1965), 816.

[174] Thompson J.S., Stmmons E.L., Crawford M.K., Severson C.D. Radiat. Res., 40 (1969), I, 70-84.

[175] SEMENov L.F. Referaty Konferentsii Ostrya luchevaya bolezni ee otdalennye prosledstviya (Extrait de la conférence «maladie aiguë des rayons et les conséquences éloignées). Sukhumi (1959), 23-25.

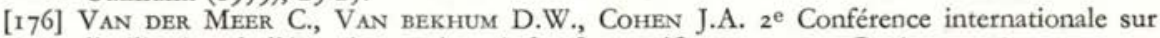
l'utilisation de l'énergie atomique à des fins pacifiques, 23, 43, Genève, 1958.

[177] Tricou B.J., Doull J., USAF. Radiat. Lab. Quarterly Progress. Report Univ., Chicago, 36 (15/7/1960), 88-103.

[178] PlzaK V., Root M., Doull J. USAF Radiat. Lab., Quarterly Progress. Report Univ. Chicago, 40 (15/7/1961).

[179] Oc'Shevskaya O.P., Jarmonenko S.P. Radiobiologiya SSSR, 9, nº I (1969), 58-6r.

[180] Ja Vorob'ev S., Gracheva A.I., Rudakov I.A., Knychev S.S. Med. Radiol., SSSR, 13, $\mathrm{n}^{\circ}$ II (1968), 64-7.

[18x] YA BoRISOvA L. Radiobiologija, SSSR, 9, $\mathrm{n}^{\circ} 2$ (1969), $246-8$.

[182] Plzak V., Root M., Doull J. USAF. Radiat. Lab. Quarterly Progress Report. Univ. of Chicago, 54 ( $15 / \mathrm{r} / \mathrm{r} 965)$.

[183] Antrpov V.V., Krasnykh I.G. Med. Radiologija, 4, I (1959), 6r-65.

[184] Savitsky J., Blood, ro, I (1955), 52-26r.

[185] MACK H., FIGGE F.H. Science, 115, 2994 (1952), 547-548.

[186] Tolkacheva E.N. Biofizika, 4, 4 (1959), 567-573.

[187] WILKINS K.H., BARNES J.H. Nature G.B., I95, 4847 (1962), II $72-73$.

[188] Haley T.J., Trumbull W.E., Cannon J.A. In: Progress in Biocbemical Pbarmacology, vol. $\mathrm{I}$, Butterworths, Washington D.C. (1965), 359.

[189] Halley T., Flesher A., Komesu N. Nature, r84 (1959), 4681, 198.

[190] Svatkov V.I. Gig. $i$ Sanit., SSSR, 33, no II (1968), 96-8.

[191] Vesely J., Gostof R., Crhak A., Sorm F., Naturforsch Z. B. Dtsch., 29, ño 3 (1969), 318-320.

[192] Chanornat P. Contribution à l'étude pharmacologique du diméthylsulfoxyde. Actions sur les gaz du sang. Thèse Doct. Vétér. Lyon, 1969 .

[193] Ashwood-Smrth M.J. Int. J. Rad. Biol., 3 (1961), 4r.

[194] Van der Meer C., Valkenburg P.W., Remmelis M. Int. J. Rad. Biol., 6 (1963), I51.

[i95] Hagemann R.F., Evans T.C., Riley E.F. Radiobiology (USA), 92, no i (1969), is 6-8. 
[196] Burnett W.T. Jr., Stapleton G.E., Morse M.L., Hollaender A. Proc. Soc. exper. biol. med., 77 (1951), 636.

[197] Косн R. In : Avances in radiobiology, G.C. de Hevesy, éd. Oliver et Boyd, Edimburg, Ecosse (1957), 170 .

[198] Bonati F., Nuvolone U. Radiobiol. Latina, I (1958), 162.

[199] Foye W.O., Marshall J.R., Mickles J. J. Pharm. Sci., 52 (1963), 406.

[200] Karrer T., Scheitling E., Siegrist H. Helv. Chim., Acta, 33 (i950), i 237.

[201] Foye W.O., Duvall R.N., Mickles J. J. Pharm. Sci., 51 (1962), I68.

[202] Foye W.O., Zaim R.H. J. Pharm. Sci., 53 (1964), 906.

[203] IrIe H. Strablentberapie, 110 (1959), 456.

[204] Alexander P., BacQ Z.M. Radiat. Res., 2 (1955), 394.

[205] Wolf V., Braun W. Arzneimittelforsch, xo (1960), 304 .

[206] Foye W.O., Kay D.H., Amin P.R. J. Pharm. Sci., USA, 57, no ro (1968), 1793-6.

[207] Carrol F.J., Dickson H.M., Wall M.E. J. Org. Chem., 30 (1965), 33 (cité dans [208]).

[208] Sugahara T.M.D., Hug O.M.D. Biological aspects of radiation protection. Ed. Igalen Shoin Ltd, Tokyo; Springer - Verlag Berlin. Heidelberg, New York, 1959.

[209] Wineman R.J., Gollis M.H., James J.C., Ponponi A.M. J. Org. Chem., 27 (1962), 4222 (cité dans [208]).

[210] Gollis M.H. Animal Progress Report $n^{\circ}{ }_{4}$, USAMRDC. Contrat no DA 49-193, MD-2109 (cité dans [208]).

[211] Maisin J.R., Mattelin G. Nature, G.B., 214, 5084 (1967), $207-208$.

$[212]$ Wang R.I.H., Hasegawa A.T. Radiat. Res., USA, 40, $\mathrm{n}^{\circ} 2$ (1969), $310-6$.

[213] Wang R.I.H., Hasegawa A.T. Radiat. Res., USA, 36, nº 2 (1968), $254,60$.

[214] Vogel H.H. Jr., Hasegawa A.T., Wang R.I.H. Radiat. Res., USA, 39, n ${ }^{\circ}$ I (1969), 57, 67.

[215] Hasegawa A.T., WANG R.I.H. Radiat. Res., USA, 31, 3 (1967), 529.

[216] Justrov V.S. Acad. Milit. Méd. Leningard. Radiobiologija, SSSR, 9, $\mathrm{n}^{\circ} 4$ (1969), $635-7$.

[217] Maisin J.R., Mattelin G., Fridman-Manduzio A., Van der Parren J. Radiat. Res., USA, $35, \mathrm{n}^{\circ}$ I (1968), 26-44.

[218] Izvekova A.V., Tumanjari M.A. Med. Radiol., SSSR, 13, no io (1968), 41-5.

[219] Szirmai E., Berkada B., Hajdukovic S., Lopez-Gonzalez J.M. Arzneimittel-FORSCH, $18, \mathrm{n}^{\circ} 5$ ( 1968$), 625-8$.

[20] Neubauer H., Graul E.H., Jacobi K.W., Ruther W., Kovacs G. Klin. Monatsble Augenbeilkde, $154, \mathrm{n}^{\circ} 3$ (1969), 453-8.

[221] Ruther W., Jacobi K.W., Kovacs G., Graul E.H., Neubauer H. Klin. Monatsbl. Augenbeilkde, $154, \mathrm{n}^{\circ} 2$ (1959), $232-8$.

[222] Zotova M.G., Borisov V.P. Radiobiologija, SSSR, 9, n ${ }^{\circ} 4$ (1969), 567-9.

[223] Klerbel F. Strablentherapie, 138, no 2 (1969), 209-12.

[224] Doherty D.G., Shapira R. J. Org. Chem., 28 (1963), 1339.

[225] Krokowski E., TaEnzer V. Strablentherapie (1966), 139-145.

[226] Visek W.J., Hung Chen Dang. Sciences News, 89 (1966), i 8.

[227] Murav'EVA L.I. Bjull. Ekesper. Biol. Med., SSSR, 33 , no s (1968), 44-46. 